\title{
Evolução da norma NBR 7188/2013: carga móvel rodoviária e de pedestres em pontes, viadutos, passarelas e outras estruturas
}

As pontes são obras de arte especiais para transpor um obstáculo natural como, mar, rio, vale e etc.. Sofrem esforços de cargas oriundas do tráfego e com o passa de sua vida útil essas cargas tendem a evoluir, devido ao crescimento populacional e aumento do carregamento dos veículos, aumentando assim os esforços. Este trabalho tem como objetivo mostrar a evolução das cargas móveis comparando a norma atual de procedimento NBR 7188 (2013), com a norma NBR 7188 (1984) e a NB06 (1960). Os parâmetros avaliados são as definições de classes, o trem-tipo que é designado com os esforços que a multidão e os veículos exercem em conjunto na ponte e o coeficiente de impacto.

Palavras-chave: Cargas móveis; Ponte; Trem-tipo.

\section{Evolution of standard NBR 7188/2013: mobile road and pedestrian loading in bridges, viadutes, walkways and other structures}

Bridges are special works of art to overcome a natural obstacle such as sea, river, valley and so on. They suffer loads from traffic loads and over their lifetime these loads tend to evolve due to population growth and increase. vehicle loading, thus increasing efforts. This paper aims to show the evolution of moving loads by comparing the current standard procedure NBR 7188 (2013), the standard NBR 7188 (1984) and NB06 (1960). The evaluated parameters are the class definitions, the type train that is designated with the efforts that the crowd and the vehicles exert together on the bridge and the impact coefficient.

Keywords: Moving loads; Bridge; Traffic Load Model.

Topic: Engenharia Civil

Reviewed anonymously in the process of blind peer.
Received: 11/11/2019

Approved: 23/12/2019
Taluane Gomes da Silva

Instituto Tocantinense Presidente Antônio Carlos, Brasil

http://lattes.cnpq.br/2111244592170164

http://orcid.org/0000-0003-1287-287X

taluane soares@hotmail.com

Douglas Freitas Augusto dos Santos

Instituto Tocantinense Presidente Antônio Carlos, Brasil

http://lattes.cnpq.br/6181125172616043

http://orcid.org/0000-0001-9306-5660

eng.prof.santos@gmail.com
Referencing this:

SILVA, T. G.; SANTOS, D. F. A.. Evolução da norma NBR 7188/2013: 'carga móvel rodoviária e de pedestres em pontes, viadutos, passarelas e outras estruturas'. Technology Science, v.2, n.1, p.53-57, 2020. DOI: http://doi.org/10.6008/CBPC2674-6425.2020.001.0009 


\section{INTRODUÇÃO}

As pontes são obras de arte especiais que estão sujeitas as cargas oriundas do tráfego e possuem a função de transpor um obstáculo natural como rio, vale etc., assim, essas cargas móveis possuem valores característicos básicos de veículos sobre pneus e ações de pedestres. Os trem-tipos ou cargas móveis, são a combinação de uma carga gerada por um veículo e as cargas distribuídas (carga de multidão).

Os trens-tipo representam as cargas móveis verticais que devem ser consideradas no projeto, na verificação e reforço das obras estruturais. De acordo a NBR 7188 (1984) trem-tipo é denominado com os esforços que a multidão e os veículos exercem em conjunto na ponte. Além disto, Fonseca Sobrinho et al. (2014) dizem que "devido à complexidade dos efeitos dinâmicos causadores do impacto é permitido assimilar a cargas móveis a cargas estáticas através da multiplicação pelo coeficiente de impacto".

\section{METODOLOGIA}

A norma NBR 7188 (2013) define os valores característicos básicos das cargas móveis rodoviárias brasileiras - 'Carga móvel rodoviária e de pedestres em pontes, viadutos, passarelas e outras estruturas'. Desde a NB-6 utiliza-se a configuração do trem-tipo com três eixos espaçados em 1,50m entre si e dimensões de $6 \mathrm{~m}$ de comprimento por $3 \mathrm{~m}$ de largura, atualmente havendo modificações nas cargas atuantes e no coeficiente de impacto.

No caso de ser construída utilizando a norma atual NBR 7188 (2013) a carga móvel rodoviária padrão TB-450 é determinada por um veículo tipo de $450 \mathrm{kN}$ com 6 rodas $P=75 \mathrm{kN}$. Um novo coeficiente de impacto que é calculado pela multiplicação do coeficiente de impacto vertical que leva em consideração o vão da estrutura, o coeficiente de número de faixas da pista e coeficiente de impacto adicional que é a respeito do tipo de material utilizado na obra. Logo, foi feito um comparativo para verificar os acréscimos gerados com a evolução das cargas móveis e mudança no coeficiente de impacto, com a NB-6 (1960), NBR 7188 (1984) e NBR 7188 (2013), conforme ilustrado na Tabela 1.

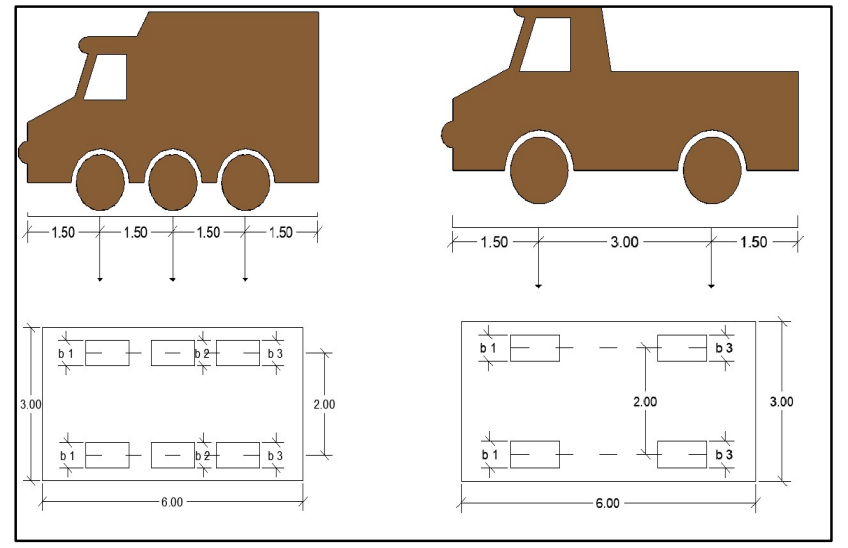

Figura 1: Trem tipo NB-6 (1960) E NBR 7188 (1984).

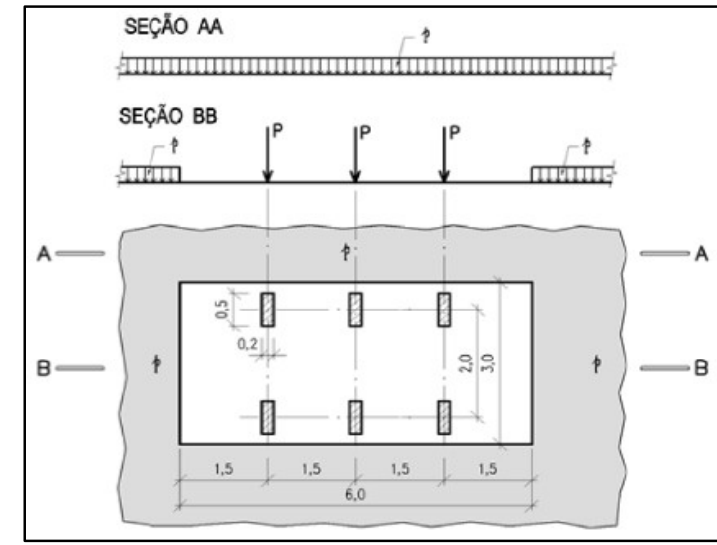

Figura 2: trem tipo NBR 7188 (2013).

Fonte: Adaptada da NBR 7188, ABNT (2013).

Tabela 1: Resumo das normas NB-6 (1960), NBR 7188 (1984) e NBR 7188 (2013).

\begin{tabular}{|l|l|l|}
\hline & Cargas Móveis & Coeficiente de Impacto \\
\hline NB-6 (1960) & $36 \mathrm{tf}$ & $\emptyset=1,4-0,007 \mathrm{~L}$ \\
\hline NBR 7188 (1984) & $45 \mathrm{tf}$ & $\emptyset=1,4-0,007 \mathrm{~L}$ \\
\hline
\end{tabular}




\begin{tabular}{|l|l|l|}
\hline NBR 7188 (2013) & $45 \mathrm{tf}$ & $\begin{array}{l}\left.C_{I V}=1,35 \text { (Para vãos }<10 \mathrm{~m}\right) ; \\
\left.C_{I V}=1+1,06 \times \frac{20}{L+50} \text { (Para vãos } \geq 10 \mathrm{~m}\right) ; \\
\end{array}$ \\
& $\begin{array}{l}C_{I A}=1,25 \text { (Para estrutura de concreto e/ou mista); } \\
C_{N F}=1-0,05(n-2)>0,9 \\
\emptyset=C_{I V} \times C_{N F} \times C_{I A}\end{array}$ \\
\hline
\end{tabular}

\section{RESULTADOS E DISCUSSÃO}

\section{NB-6/60}

Utilizando o trem-tipo para a ponte classe $36 \mathrm{tf}$ de acordo com a NB-6 (1960) se encontra os carregamentos dos esforços atuantes na estrutura com veículo tipo e multidão uniformemente distribuída constantemente (Figura 1).

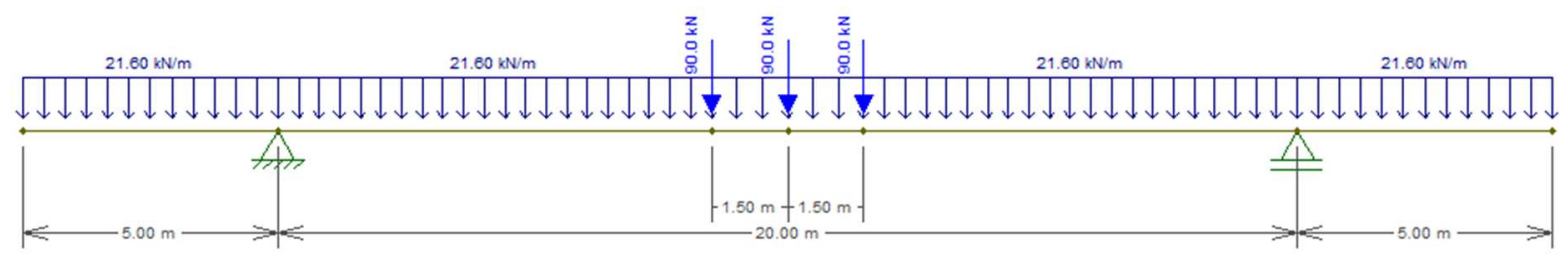

Figura 3: Cargas móveis atuantes na estrutura.

Já o seu coeficiente de impacto é em função do vão e obtém-se o valor de 1,26 e 1,365 para o vão de $20 \mathrm{~m}$ e os balanços de $5 \mathrm{~m}$, respectivamente. As cargas atuantes no trem-tipo através das envoltórias geraram os esforços cortantes máximos de $Q_{+}=654,2 \mathrm{KN}$ e $Q=6544,2 \mathrm{KN}$ e momentos máximos de $M_{+}=2894,7 \mathrm{KN} \cdot \mathrm{m}$ e $M=1658,5 \mathrm{KN} \cdot \mathrm{m}$

\section{NBR $7188(1984)$}

Utilizando a ponte classe 45 tf de acordo com a NBR 7188 (1984) se encontra os carregamentos dos esforços atuantes na estrutura com veículo tipo e multidão uniformemente distribuída (Figura 2).

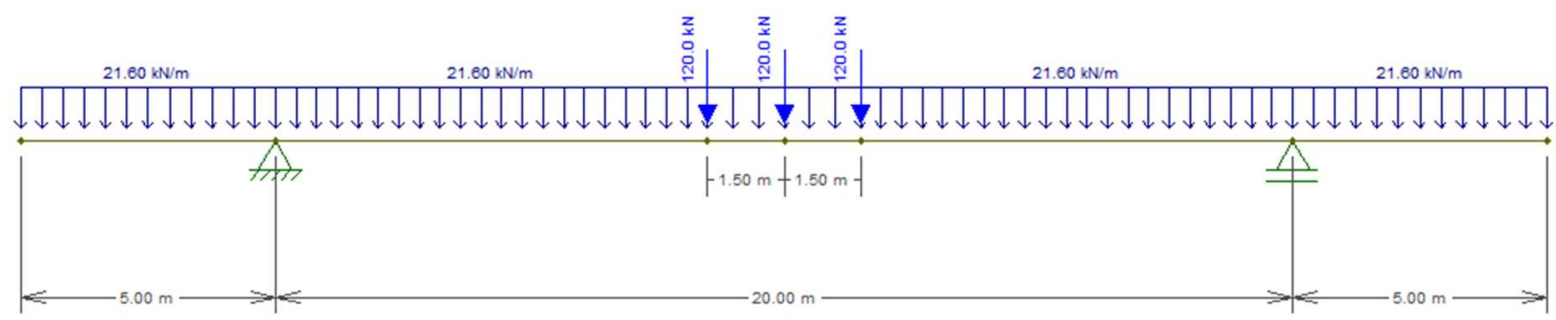

Figura 4: Cargas móveis atuantes na estrutura.

Já o seu coeficiente de impacto é em função do vão e se obtém os mesmo valores obtidos com anteriormente visto que a expressão é a mesma da norma NB-6 (1960). As cargas atuantes no trem-tipo através das envoltórias geraram os esforços cortantes máximos de $Q_{+}=770 \mathrm{KN}$ e $Q_{-}=-770 \mathrm{KN}$ e momentos máximos de $\mathrm{M}_{+}=3402 \mathrm{KN} \cdot \mathrm{m}$ e $\mathrm{M}_{-}=2088,5 \mathrm{KN} \cdot \mathrm{m}$.

\section{NBR 7188 (2013)}

Utilizando o trem-tipo para a ponte classe 45 tf de acordo com a NBR 7188 (2013) se encontra os carregamentos dos esforços atuantes na estrutura com veículo tipo e multidão uniformemente distribuída 
constantemente (Figura 2). A carga concentrada ' $Q$ ', em kN, e a carga distribuída ' $q$ ', em kN/m², são os valores da carga vertical móvel aplicados no nível do pavimento, iguais aos valores característicos majorados pelos Coeficientes de Impacto Vertical (CIV), do Número de Faixas (CNF) e de Impacto Adicional (CIA), conforme a equação 2 .

$$
\mathbf{Q}=\mathbf{P} \times \mathbf{C I V} \times \mathbf{C N F} \times \mathbf{C I A} \quad \text { Equação } 1
$$

Utilizando as fórmulas supramencionadas se calcula o coeficiente de impacto com a multiplicação, além disso chega-se nos valores para o $C_{I V}=1,35$ (estrutura em balanço) e $C_{I V}=1,303$ (para $L=20 m$ ), $C_{N F}=1,0$ e $C_{1 A}=1,25$ (para concreto) e substituindo encontra-se o valor do coeficiente de impacto de 1,69 (para balanço) e 1,63 (para vão). As cargas atuantes no trem-tipo geraram os esforços cortantes máximos de $Q_{+}=950,6 \mathrm{KN}$ e $Q=-950,6 \mathrm{KN}$, e os momentos máximos foram $\mathrm{M}_{+}=4401 \mathrm{KN}$.m e $\mathrm{M}_{-}=2585,7 \mathrm{KN} . \mathrm{m}$.

\section{Evolução das Cargas}

Na Figura 6 verifica-se a evolução dos esforços de acordo com a norma. Nota-se que ocorre um aumento com a consideração do TB36 que era a carga máxima utilizada pela NB6 (1960) para o TB45 da NBR 7188 (1984), que foi cerca de $15 \%$ para o esforço cortante, $23 \%$ para o momento positivo e $21 \%$ para o momento negativo. Além disso, a mudança se torna maior quando consideramos a norma mais recente NBR 7188 (2013), cerca de 31\% para o esforço cortante, 34\% e 36\% para o momento positivo e negativo, respectivamente. Fato explicado pela alteração do coeficiente de impacto, que pela norma atual, é mais abrangente.

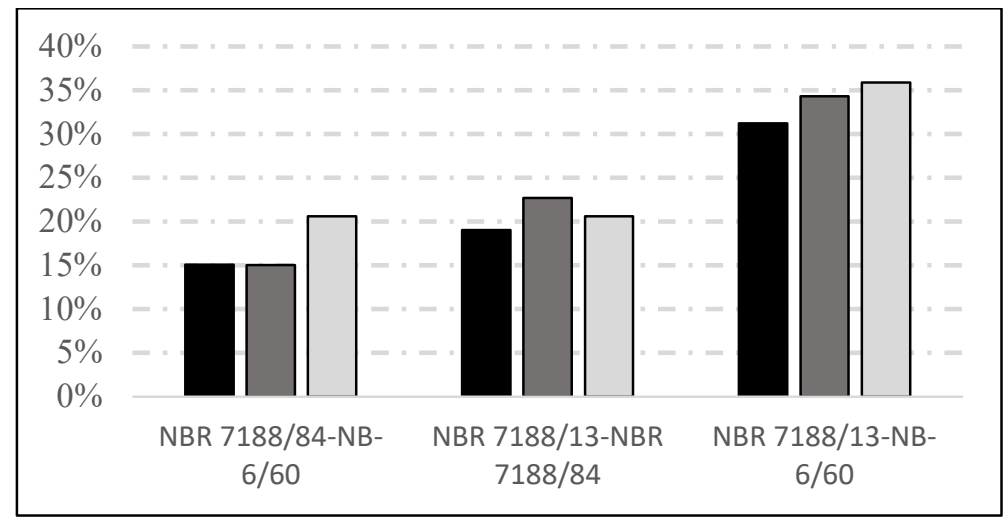

Figura 6: Comparativo em percentual dos esforços.

\section{CONCLUSÕES}

Há um acréscimo de cargas geradas ao longo dos anos desde a NB-6 (1960) e isso mostra a importância de se fazer as inspeções para que possa ter manutenções e caso necessário reforçar as pontes antigas. A grande maioria das pontes brasileiras foram construídas bem antes da norma vigente NBR 7188 (2013). A exemplo temos a ponte de Porto Nacional, localizada no estado do Tocantins, município a 60km da capital Palmas. Esta ponte foi construída na década de 70, utilizando como base a norma vigente da época, ou seja, a NB6 (1960). 


\section{REFERÊNCIAS}

ABNT. Associação Brasileira de Normas Técnicas. NB-6: Carga móvel em ponte rodoviária e passarela de pedestre. Rio de Janeiro: ABNT, 1960.

ABNT. Associação Brasileira de Normas Técnicas. NBR 7188: Carga móvel rodoviária e de pedestre em pontes, viadutos, passarelas e outras estruturas. Rio de Janeiro: ABNT, 1984.
ABNT. Associação Brasileira de Normas Técnicas. NBR 7188 Carga móvel rodoviária e de pedestre em pontes, viadutos, passarelas e outras estruturas. Rio de Janeiro: ABNT, 2013.

FONSECA SOBRINHO, B.; BRITO, M. A. N.; SANTOS, I. C.; SILVA, R. S. Y. C.; EMIDIO SOBRINHO, B.. Efeitos da Mudança da NBR 7188:2013 nos Projetos de Pontes. Estudo de Caso: Projeto de Recuperação da Ponte sobre o Rio Correias na BR 101/SC. In: CONGRESSO BRASILEIRO DE PONTES E ESTRUTURAS. Anais. Rio de Janeiro, 2014.

A CBPC - Companhia Brasileira de Produção Científica (CNPJ: 11.221.422/0001-03) detém os direitos materiais desta publicação. Os direitos referem-se à publicação do trabalho em qualquer parte do mundo, incluindo os direitos às renovações, expansões e disseminações da contribuição, bem como outros direitos subsidiários. Todos os trabalhos publicados eletronicamente poderão posteriormente ser publicados em coletâneas impressas sob coordenação da Sapientiae Publishing, da Companhia Brasileira de Produção Científica e seus parceiros autorizados. Os (as) autores (as) preservam os direitos autorais, mas não têm permissão para a publicação da contribuição em outro meio, impresso ou digital, em português ou em tradução. 